\title{
ON THE ELECTROWEAK ONE-LOOP CORRECTIONS
}

\author{
V.A. Novikov \\ ITEP, 117259, Moscow, Russia \\ L.B. Okun *) \\ Theoretical Physics Division, CERN \\ CH 1211 Geneva 23, Switzerland \\ and \\ M.I. Vysotsky \\ ITEP, 117259, Moscow, Russia
}

\section{ERRATUM}

For unknown circumstances, some of the equations of this paper were partly scratched from the file and were not correctly printed. Please, find hereafter the correct equations.

*) Permanent address: ITEP, Moscow, 117259, Russia.

CERN-TH.6538/92/ERRATUM

ITEP-67/92

August 1992 
$\underline{\text { Page } 13}$

$$
\begin{aligned}
T^{A}(t)= & \frac{2}{3}-\frac{8}{9} s^{2}+\frac{16}{27} s^{4}-F_{t}^{\prime}(t)+\left(\frac{32}{9} s^{4}-\frac{8}{3} s^{2}-\frac{1}{2}\right) \times \\
\times & \left(\frac{4}{3} t F_{t}(t)-\frac{2(1+2 t)}{3} F_{t}^{\prime}(t)\right)
\end{aligned}
$$

Page 31

$$
\begin{aligned}
& H_{A}(h)= \frac{h c^{2}}{h-c^{2}} \ln \left(\frac{h}{c^{2}}\right)-\frac{8 h}{9(h-1)} \ln (h)+\left(\frac{4}{3}-\frac{2}{3} h+\frac{2}{9} h^{2}\right) F_{h}(h)- \\
&-\left(\frac{4}{3}-\frac{4 h}{9}+\frac{h^{2}}{9}\right) F_{h}^{\prime}(h)-\frac{h}{18}+0.68 \\
& H_{R}(h)=-\frac{16 \pi}{3 \bar{\alpha}} c^{2} s^{2}\left[\frac{\left(c^{2}-s^{2}\right)}{c s} \Pi_{Z_{\gamma}}\left(m_{Z}^{2}\right)+\Pi_{\gamma}\left(m_{Z}^{2}\right)-\Pi_{Z}\left(m_{Z}^{2}\right)+\right. \\
&\left.+\Pi_{W}(0)+2 \frac{s}{c} \Pi_{\gamma Z}(0)\right]-\operatorname{div} H_{R}(h) \cdot \\
&\left.* H_{R}(h)=-\frac{h}{18}+\frac{c^{2}}{1-c^{2} / h} \ln +\frac{4}{9} h+\frac{1}{9} h^{2}\right) F_{h}(h)+ \\
& \quad+\frac{h}{1-h} \ln h+0.03 . \\
& \text { div } H_{R}(h)= \\
&\left.\quad \frac{7}{6} \frac{s^{2}}{c^{2}}\right)+\frac{2}{9}\left(c^{2}-s^{2}\right)\left(1+30 c^{2}\right)+4 c^{2} s^{2}+\frac{4}{3} c^{2}\left(7 s^{2}-\frac{25}{6}+\right. \\
&
\end{aligned}
$$

